\title{
Inmersos en el ecosistema tropical - viaje por América Latina y algunas arquitecturas del trabajo en red $^{1}$
}

Rosa Apablaza Valenzuela*

http://dx.doi.org/10.22409/poiesis.1829.093112

RESUMEN: Este texto surge a partir de un viaje que realicé en febrero y marzo 2012 por Perú, Bolivia, Argentina, Brasil y Ecuador. Entre 2006 y 2011, realicé a través del proyecto Desislaciones, un proceso de investigación, difusión y vinculación de colectivos, organizaciones sociales y agrupaciones que trabajaban de forma colaborativa en la región. El proyecto fue desarrollado principalmente desde España, país donde residí durante el mismo período. Si bien durante esos cinco años, fui articulando una serie de contactos presenciales y actividades con algunos colectivos o integrantes de ellos, el motivo del viaje surgió por la necesidad de entrar en contacto directo y experimentar de primera mano intercambios subjetivos y formas de organización que venía investigando desde la distancia. Aquí relato mi experiencia en Brasil, específicamente en Fiar 3, Festival de Intervenções Artísticas do Recôncavo Bahiano.

PALABRAS CLAVE: colectivos, intercambios subjetivos, formas de organización, FIAR 3

ABSTRACT: This text comes from a trip I made in February and March 2012 in Peru, Bolivia, Argentina, Brazil and Ecuador. Between 2006 and 2011, I carried out through the Desislaciones project, a research, dissemination and linking process of collectives, social organizations and groups that worked collaboratively in the region. The project was developed mainly from Spain, country where I resided during the

\footnotetext{
* Rosa Apablaza Valenzuela es artista visual, performer, gestora cultural y escritora de textos críticos sobre arte. Licenciada en Artes por la Universidad de Chile. Creadora del proyecto Desislaciones (2006/2011), co-creadora de los proyecto Trans Acciones Utópicas (2016, junto a Centro Rural de Arte, Argentina) y de Ecossistema Tropical 2.0 (2012, Junto a Felipe Brait, Brasil). Ha sido becada para realizar diversas residencias para artistas, tanto en Latinoamérica como en Europa. Ha coordinado y participado en diversos proyectos, exposiciones colectivas y encuentros internacionales y ha publicado en revistas, catálogos y medios independientes de Chile, Argentina, Brasil, México, Colombia, Cuba y España. Entre 2012 y 2014 fue columnista en ArteyCritica.org. Actualmente es miembro del equipo editorial de Los Libros de La Mujer Rota (Chile).
} 
same period. Although during those five years, I was articulating a series of face-to-face contacts and activities with some groups or members of them, the reason for the trip arose from the need to come into direct contact and to experience firsthand subjective exchanges and forms of organization that I had been investigating from a distance. Here I tell my experience in Brazil, specifically in Fiar 3, Festival of Artistic Interventions of Recôncavo Bahiano.

KEYWORDS: collective, subjective exchanges, forms of organization, FIAR 3

La información disponible es abrumadora: todo un universo de ideas, imágenes y textos que no cesa de expandirse en múltiples direcciones. Cada elemento posible tiene muchas capas de significado en las que podemos buscar y cada elemento a su vez se haya conectado potencialmente con otros muchos, tal vez pertenecientes a categorías muy distintas.

-- Ricard Solé, Redes Complejas. Del genoma a internet.

\section{En busca del ecosistema tropical}

Este texto surge a partir de un viaje que realicé en febrero y marzo 2012 por Perú, Bolivia, Argentina, Brasil y Ecuador. Entre 2006 y 2011, realicé a través del proyecto Desislaciones², un proceso de investigación, difusión y vinculación de colectivos, organizaciones sociales y agrupaciones que trabajaban de forma colaborativa en la región. El proyecto fue desarrollado principalmente desde España, país donde residí durante el mismo período. Si bien durante esos cinco años, fui articulando una serie de contactos presenciales y actividades con algunos colectivos o integrantes de ellos, el motivo del viaje surgió por la necesidad de entrar en contacto directo y experimentar de primera mano intercambios subjetivos y formas de organización que venía investigando desde la distancia.

Aquí relato mi experiencia en Brasil, específicamente en Fiar 3, Festival de Intervenções Artísticas do Recôncavo Bahiano ${ }^{3}$. 


\section{Fiar Bahía 3: ecosistema tropical expandido.}

Esta etapa fue una de las principales motivaciones para realizar el viaje: el encuentro FIAR Bahía 3, que se llevó a cabo entre el 29 de febrero y el 3 de marzo de 2012, organizado por Tininha Llanos y José Balbino, fundadores de Casa da Alegría 4 .

Se realizó entre las localidades de São Félix y Cachoeira, ciudades del recôncavo bahiano, separadas por el río Paraguaçú, unidas por el Puente Imperial Pedro II, que por su ubicación estratégica, son de extrema significancia en la fundación de Brasil, su tradición esclavista y el proceso de independencia de Bahía.

Aquí tuve la oportunidad de compartir y experimentar un proceso de intercambio con colectivos brasileños de distintas ciudades. Algunos nuevos y otros con los cuales venía tejiendo lazos desde el 2007, mediante los proyectos Desislaciones y Residencia Temporal ${ }^{5}$.

El encuentro, cuya programación estuvo compuesta por la participación de artistas y colectivos, y la realización de performances, talleres, charlas, proyecciones, intervenciones colectivas y experimentaciones que promovían acciones integradas a la atmósfera local, fue una experiencia de un valor incuantificable, en tanto espacio de compartimiento, diálogo, comunidad temporal y pertenencia, producción de subjetividades y afectos y también por todas las posibilidades de encuentro y colaboración que me brindó para los días siguientes en Brasil, para entender ese contexto y para crear fuertes lazos de trabajo y amistad que perduran hasta el día de hoy.

En palabras mencionadas en el blog de FIAR por la curadora y productora ejecutiva del festival Tininha Llanos:

Fiar es hacer red, y Fiar 3 se propone como un festival de intervenciones en el cual los residentes son generadores de redes artísticas en sus localidades. Justamente por entender que en otro momento esos grupos ya realizaron un mapeamiento de grupos y artistas individuales en sus territorios de origen, correspondiendo al festival proporcionar un espacio de encuentro de redes, en la construcción de un contexto más amplio de producción y circulación artística.

Cuatro años después, en 2016, durante Ecosistema Tropical 2.06 (proyecto que realizamos junto a Felipe Brait y nueve colectivos brasileños en cuatro ciudades de Brasil; São Paulo, Salvador, Río de Janeiro y Belem), entendí con precisión los antecedentes históricos de una red de artistas que trabajaban con intervenciones urbanas en Brasil, de los cuales varios participaron de FIAR. Lo cual se explica con la consecución histórica de los siguientes hechos: 
Alrededor del año 2000, surgió en el contexto artístico brasileño una gran diversidad de creadores que proponían otras prácticas, que dentro de los circuitos comerciales y estatales no tenían cabida, principalmente vinculadas al trabajo colectivo y al espacio público. Existió entre ellos una sincronía que se fue dando de forma espontánea por los propios cambios históricos. Uno de los hechos históricos determinantes fue la relación que establecieron con el Movimiento de Moradía, en el cual en palabras de Felipe Brait los colectivos "vieron una vía para la politización y radicalización de sus prácticas."

Este lo explica como:

el Movimiento Social de Moradía ofreció un aparataje político a los grupos artísticos al mismo tiempo que los colectivos ofrecieron una dimensión estética al movimiento social, uno de los hechos emblemáticos en este sentido fue la colaboración que existió de parte de varios colectivos de arte para resistir el desalojo de la Ocupação Prestes Maia en Sao Paulo.

Por lo tanto, al referirme al centro de las cuestiones desarrolladas en FIAR no puedo dejar de mencionar sus objetivos implícitos: poner en valor el trazado de los caminos recorridos anteriormente entre los colectivos, revivir un modelo de organización grupal que desde años atrás venían tejiendo desde un espacio afectivo. Tal como dice Felipe "un agenciamiento biopolítico que parte de los afectos, nosotros nos organizábamos, mirando unos las caras de los otros, ahí decidíamos un trabajo e íbamos para la calle, ¿por qué a la calle? Porque las galerías, museos y centros culturales no aceptaban aquello que nosotros queríamos expresar en cuanto a arte, por eso también el uso del espacio público".

Otro antecedente importante es que muchos de estos artistas que aún eran estudiantes, comenzaron a usar las posibilidades que tenían para encontrarse con los distintos nodos emergentes distribuidos por Brasil. Una de ellas eran los encuentros estudiantiles, tales como congresos o salones nacionales de estudiantes. Estos encuentros fueron fundamentales para que los distintos colectivos que emergían se fueran encontrando y fortaleciendo, lo cual derivó en que entre algunos crearan su propio salón de arte que fue el I Salão de Maio, realizado en Salvador de Bahía en 2004, que después tuvo una II versión en 2005, ambos enfocados en intervenciones urbanas. En estos primeros encuentros lo más importante era estar juntos, crear un sentido de pertenencia y la construcción de una escena que otorgara legitimidad a estas nuevas prácticas para poder salirse de los espacios de estandarización y normatividad. 


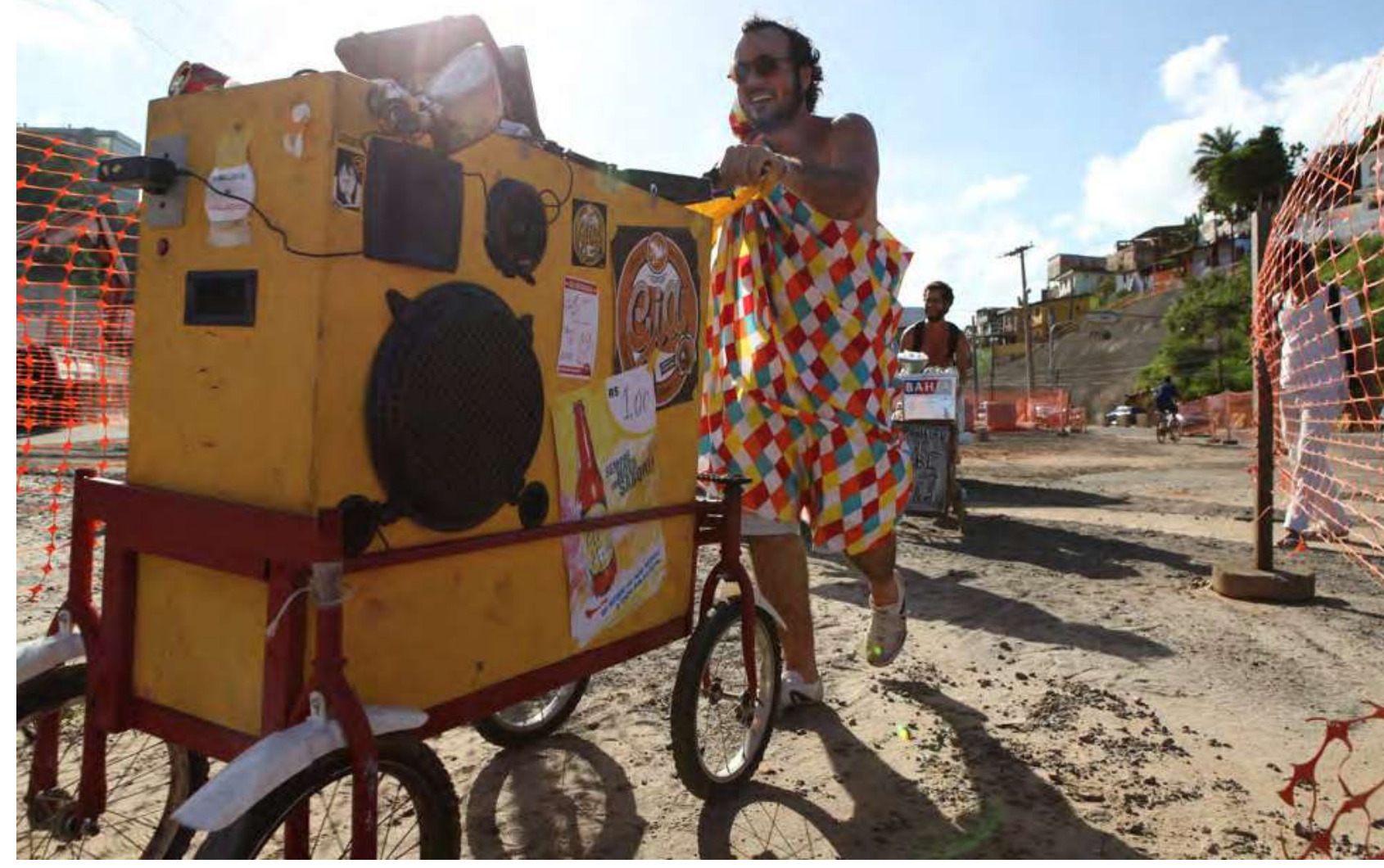

Intervención "Descascando o abacaxi" de OPA! + GIA durante Me Dê Motivos. residência processual colaborativa na Cidade de Salvador, Bahia.

(Foto: cortesía de Opavivará)

Un hecho importante de mencionar es que este encuentro, donde GIA (Grupo de interferencia ambiental) estaba invitado, coincidió con la realización de la residencia artística ME DÊ MOTIVOS $\rightarrow$ OPA!+GIA que ese mismo año se adjudicó un edital de la red nacional de Artes Visuales de FUNARTE, motivo por el que el colectivo Opavivará estaba en el encuentro.

Entonces, puedo decir que lo que primó en FIAR fue establecer a través de acciones, relaciones críticas con el territorio, inmersiones, interferencias, el uso de la ciudad como campo de acción, pero por sobre todo el fortalecimiento de ese tejido que había comenzado alrededor de los años 2000. 


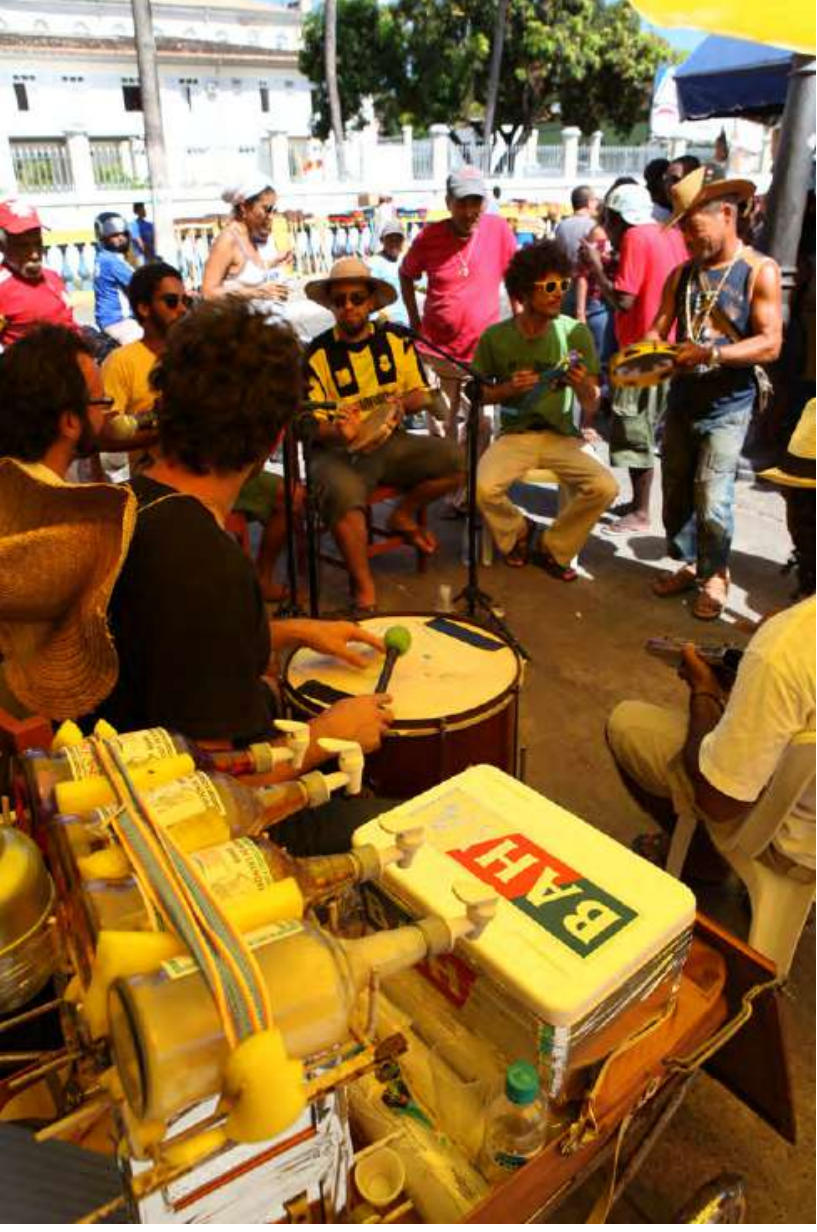


Cerca de las 16 hrs., comenzaron una serie de charlas en el Centro Cultural Dannemann en São Félix, las cuales tenían por título "As Redes Colaborativas de Arte". Los participantes fueron: Patricia Francisco (RS), Milena Durante ${ }^{8}$ - EIA (SP), Rosa Apablaza - Desislaciones (Chile) y Luis Parras - GIA e PIA (SP/BA). De forma simultánea a esto, rádio amnésia dirigida por Sérgio Melo, comenzó un programa radial donde se transmitieron las charlas. Por mi parte expresé la necesidad de renovar y experimentar nuevas formas de organización política respecto al trabajo en red y la importancia del uso de herramientas tecnológicas que permitiesen mayor acercamiento entre los diversos nodos de creación artística/activista en Latinoamérica.

El día siguiente, $1^{\circ}$ de marzo, comenzó aproximadamente a las 10 de la mañana con el artista Ricardo Brazileiro quien realizó la acción "3CO" en el Jardim Grande de Cachoeira la cual consistió en

una intervención urbana interactiva que colectaba datos ambientales en tiempo-real y actuaba creando un ecosistema híbrido que reaccionaba con las intensidades del cotidiano urbano. Las reacciones eran acopladas en servomotores que accionaban unos paraguas equipados con sensores analógicos y digitales capaces de sentir informaciones como temperatura, intensidad de luz y ruidos, señales sonoras, gases y contaminación. ${ }^{10}$

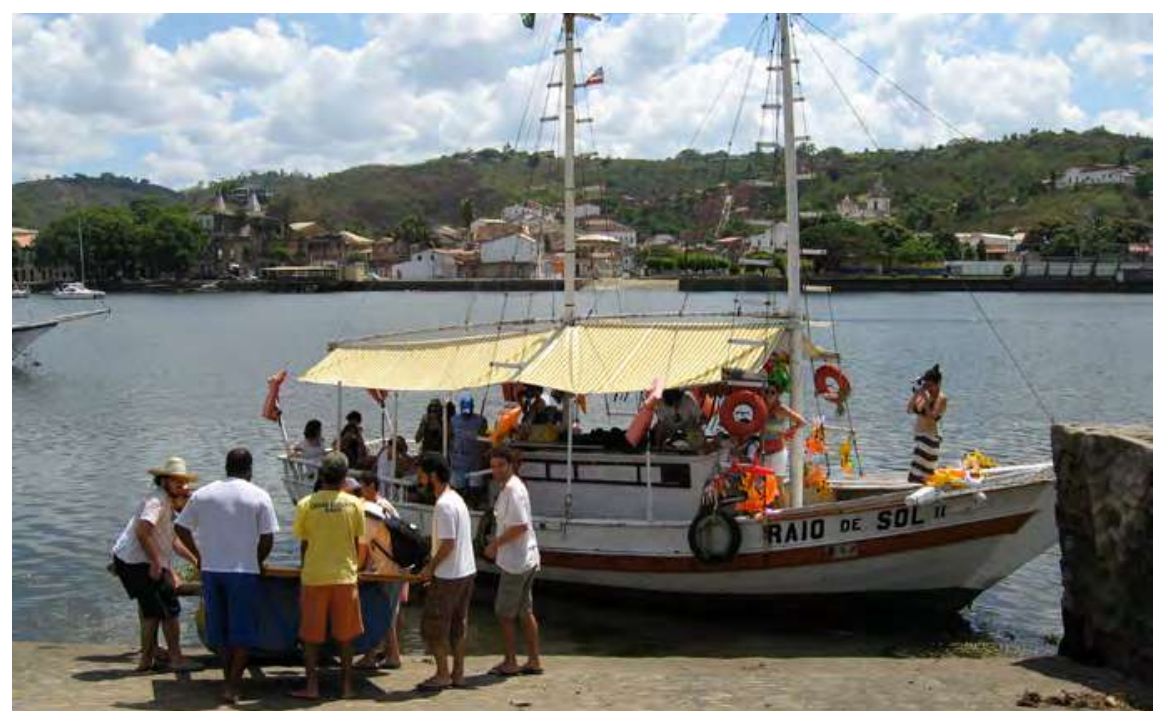

Flutuador.

Acción Flutuador de Colectivo GIA en colaboración con OPA! en Río Paraguaçú. 
Después de esta intervención volvimos al Centro Cultural Dannemann donde se realizaron un bloque de charlas llamadas "intervenções urbanas: poéticas e ativismo - proposta para um fazer junto," donde participaron Cristiano Piton (GIA-BA), WG (PI), Marcelo Terça-Nada (POROMG) y Felipe Brait (Frente de Três de Fevereiro-SP). A las 18 hrs. en el mismo lugar se realizó el lanzamiento del libro Intervalo, Respiro, pequenos delocamentos - ações poéticas do Poro. A las 20 hrs. en el mismo tuvo lugar una "Palestra Show"11 dirigida por los mestres puerto alegrenses Paraqueda y Paulo Romeu, acompañados por el mestre Valmir, de a Irmandade da Boa Morte y del compositor y profesor Cristiano Figueiró. Esta actividad consistió en la presentación del proyecto Samba e Raiz Africana ${ }^{12}$ realizado en Puerto Alegre (aprobado por FUNARTE, dentro del premio Interações Estéticas, residencias artísticas em pontos de cultura) y una presentación musical entre ellos en conjunto con distintos músicos participantes del encuentro.

El 2 de marzo comenzó cerca de las 7 de la mañana, mediante un debate libre durante un viaje en barco descendiendo por el Río Paraguaçú, una experiencia liberadora de pertenencia, viaje colectivo y comunidad. La posibilidad de este viaje estaba abierta al público y entre quienes se adhirieron estaba el escritor Marcio Junqueira. Durante esta jornada el Colectivo GIA realizó en el mismo río la acción Flutuador que muy bien lo precisa la descripción publicada en el blog de FIAR:

Objetivamente, el flotador es una estructura como un 'deck' flotante, anclado en una playa pública, al cual puede tener acceso cualquier persona y que esa persona pueda darle el uso que desee. Es decir, el objetivo también de este proyecto es plantear cuestiones acerca de la libertad de utilización de los espacios públicos ya que ninguna regla existe en este ambiente, que es temporalmente gestionado por las personas en el instante en que lo ocupan, además que la distancia física de la tierra (playa) posibilita muchas oportunidades de actuación y libertad. ${ }^{13}$

El 3 de marzo se realizó el proyecto Calón-a-quilo, de CAMBANA ${ }^{14}$, producto de una investigación realizada desde 2010 por Maicyra León y Márcio Lima en conjunto con gitanos en sus campamentos en el Recôncavo Baiano. Consistió en acciones en la feria de la ciudad, entre las tiendas de los feriantes y de forma itinerante, realizadas por artistas que dialogaron con aspectos de la experiencia en las comunidades. También proyectaron un documental sobre el proyecto.

Entre las 14 y 19hrs. realizamos diversas Intervenções Coletivas en Cachoeira. 
El mismo día, GIA, integrado por Luis Parras, Everton Marco, Tiago Ribeiro, Mark Dayves, Cristiano Piton, Tininha Llanos y Ludmila Britto nos deleitaron con SAMBAGIA ${ }^{15}$ la acción comenzó con un recorrido con un carrinho por la feria de Cachoeira y después con una estación más extensa frente a un bar donde estuvimos bailando, cantando y bebiendo. El centro de la fiesta fue la musa Michelle Mattiuzzi, artista de performance. Me viene esta imagen a la cabeza: el mundo se estaba derrumbando y nosotros estábamos sambando encima de las ruinas, a veces el único lugar de fuga.

Terminamos con música en vivo y baile en homenaje al género afrobeat en el Centro Cultural Dannemann por el artista sonoro y productor Edbrass Brasil, junto a la manipulación de videos en tiempo real por parte de Jarbas Jácome.

El encuentro nos devolvió la consciencia de okupar y apropiarnos del espacio público y experimentar instancias de comunidad que, como diría Raúl Zibechi en su libro "Autonomías y emancipaciones: América Latina en movimiento (2007)", contiene en sí misma un poder curativo. Experimentamos lo colectivo como una experiencia de desalienación, y vivimos de primera mano la soltura y cooperativismo presente en la extensa tradición de trabajo colectivo que existe en Brasil.

En las cosmovisiones tradicionales no existe separación entre salud y forma de vida, o sea, comunidad. Por eso "la salud de los individuos en cuanto cuerpos físicos, depende, básicamente de la salud de la comunidad". (MALDONADO, 2003) El concepto curativo de la medicina indígena forma parte del concepto curativo de esa sociedad, y se asienta, por un lado, en una tupida red de relaciones sociales de reciprocidad: minga o trabajo comunitario, asambleas y fiestas colectivas: espacios para "liberar armoniosamente el subconsciente, tanto el individual como el colectivo (RAMÓN, 1993, p. 329)." (ZIBECHI, 2007, p. 39)

\section{Herramientas tecno-políticas}

Nuestra sociedad en red hace cada vez más posible que pequeños cambios desencadenen grandes efectos. Cada uno de nosotros, empleando adecuadamente lo que la red le ofrece, puede participar en

la historia y su devenir. (SOLÉ, 2009, p. 48)

Durante el encuentro, difundí, como herramientas tecno-políticas, los proyectos N-1 y su nodo latinoamericano AnilloSur ${ }^{16}$, que ponen a disposición una serie de herramientas mediante una plataforma de código abierto para el intercambio de contenidos, la organización y acción de 
grupos sociales y el aprendizaje colectivo. N-1 ha sido creado por Lorea, federación de servidores libres"17, que se define como un "semillero" de redes sociales sobre un campo de experimentación ${ }^{18}$. Hablamos de la importancia de que velemos por la seguridad de la información que compartimos o los datos que subimos a una determinada plataforma. En gran medida porque todos los deseos y flujos de información que existen tras nuestro "deseo de comunidad" son y podrían seguir siendo cooptados por el mercado cultural, el mayor depredador dentro de nuestro ecosistema tropical, por boicots a la cultura libre y a nuestras prácticas subjetivas.

El trabajo en red requiere de un soporte que albergue su desarrollo; un medio o hábitat que permita la interacción entre los nodos, el almacenamiento de datos o la generación y compartimiento de servicios en una comunidad. Estos medios van variando, determinados por el tipo de relaciones en la interacción, su emergencia en tanto red y diversas fugas que dependen

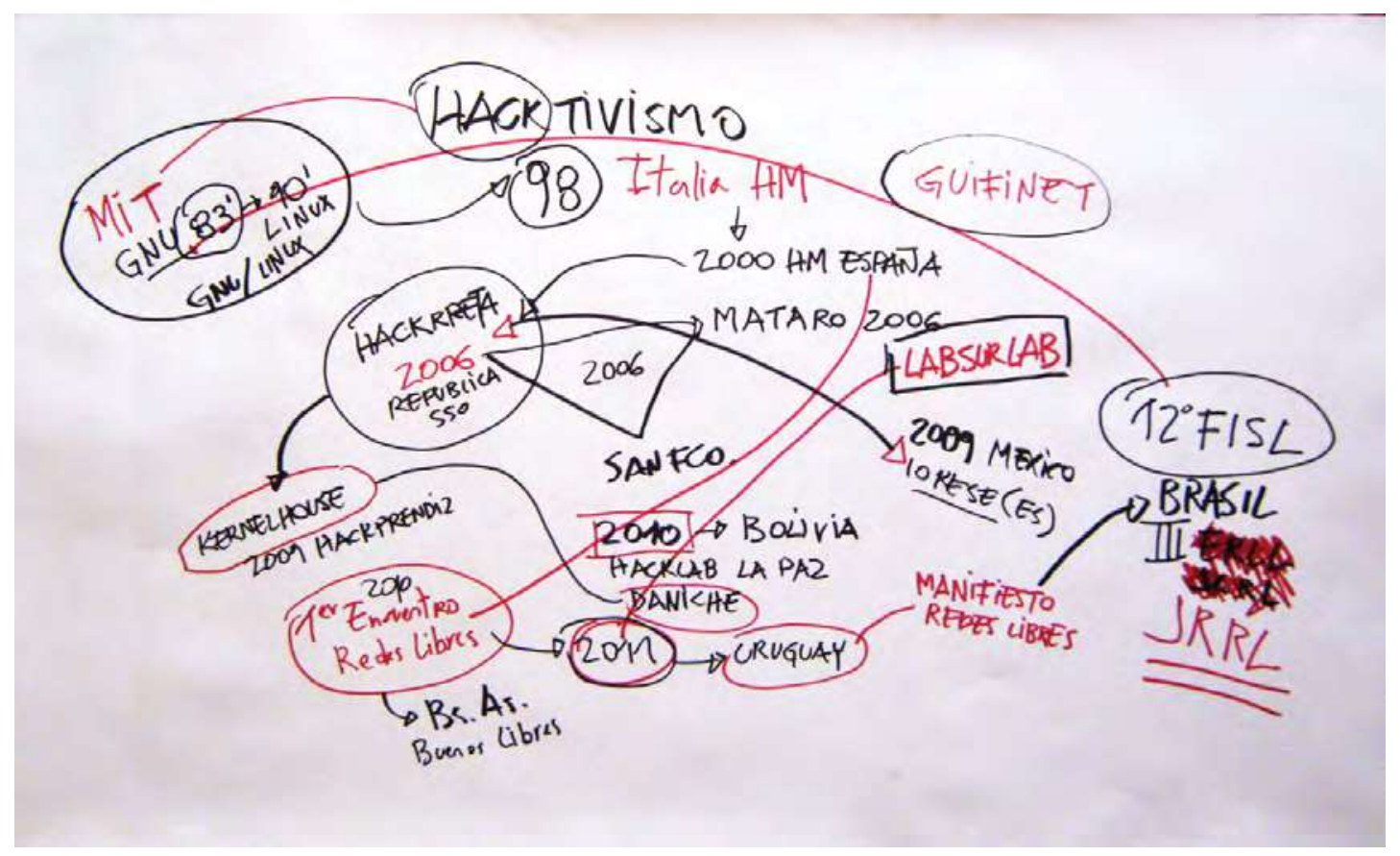

Mapa sobre el hacktivismo en América Latina, realizado por Héctor Capossiello, hacker, mediactivista chileno y parte del grupo de administradores de N-1 y AnilloSur. 
de las necesidades de la red y su evolución. En las redes que funcionan de forma presencial, el hábitat, podría ser algo tan sencillo como un espacio físico. Otras redes combinan espacios virtuales y físicos, mediante actividades, como encuentros, seminarios, etc. y en otros casos, mediante hackerspaces que integran hacklabs o laboratorios hacker o makerspaces (espacios o comunidades donde personas con intereses principalmente basados en tecnologías libres y procomún se reúnen, socializan, comparten conocimientos y colaboran en la producción de proyectos) o simplemente espacios informales compartidos.

Existen diversas herramientas tecno-políticas que nos permiten el trabajo en red, a través de metodologías de trabajo participativo. Una de las acepciones que más acertadas me parece para explicar tanto estas herramientas como el trabajo colaborativo, es la que propone Transductores ${ }^{19}$ al explicar el concepto que da nombre a su proyecto:

son dispositivos que traducen, median y producen nuevas energías, pero sin demarcar su orientación o su valor, sino esperando que el cuerpo donde se inscribe el proceso de transformación se adapte y reinvierta sus capacidades e intereses en multiplicar esta energía. [...] Desde la perspectiva de los movimientos sociales, los estilos transductores median y negocian los objetivos políticos de un movimiento, facilitando que emerjan energías diferentes según las diversas metas y modos de actuación, de tal modo que se producen nuevos desbordes y evoluciones inesperadas. Los transductores trabajan con las sinergias de cada movimiento y situación social, abriendo nuevas posibilidades de cambio, más complejas y globales, creando situaciones peculiares u operando como disparadores, al mismo tiempo que generan el intercambio de conocimientos y de estilos de vida entre los agentes implicados). (TRANSDUCTORES, 2009, p. 17-18)

Fiar fue una experiencia de inmersión en el tramado conformado por colectivos de arte/política brasileños, que nos otorgó una dimensión bastante precisa sobre las dinámicas del trabajo colaborativo en el país, que sin duda no escapa a aspectos culturales y sociales predominantes en Brasil, tales como la experiencia de la celebración, la fiesta y okupación del espacio público, en tanto experimentación colectiva de insurrección y la práctica del grupo como modelo paleolítico primario y radical, mencionado en la Taz de Hakim Bey en contraposición a la estructura cerrada de la familia como núcleo.

La familia queda cerrada, por lo genético, por la posesión machista de la mujer y los niños, y por la jerárquica totalización de la sociedad agrícola-industrial. El grupo en cambio está abierto. No a todos, por supuesto, pero sí a los grupos de afinidad y a los iniciados que se comprometen por 


\section{ME DÊ MOTIVOS. Salvador de Bahía - Compartiendo con OPA! + Gia}

Después de FIAR 3, junto a Felipe Brait y Michelle Mattiuzzi, tuvimos el honor de compartir, en casa de Dalila Pinheiro, con los colectivos OPAVIVARÁ! + GIA durante una experiencia una semana que llamaron ME DÊ MOTIVOS. RESIDÊNCIA PROCESSUAL COLABORATIVA NA CIDADE DE SALVADOR, BAHIA. ${ }^{21}$

Guiados por ellos recorrimos la ciudad, entramos a espacios en desuso, charlamos acerca de las diferencias en cuanto a prácticas de okupación de España y Brasil, donde en este último país eran más escasos los espacios okupados que arrancaban de la marginalidad, y que se conformaban como organizaciones en contra de la especulación inmobiliaria, como formas de gestión colaborativa de espacios de vivienda o como centros sociales (situación que en la actualidad ha cambiado bastante).

Respecto a esto, ahondamos en una entrevista que realizamos durante nuestra estancia en Bahía a Pedro Victor Brandão, en ese entonces integrante de Opavivará!, quien realizó un

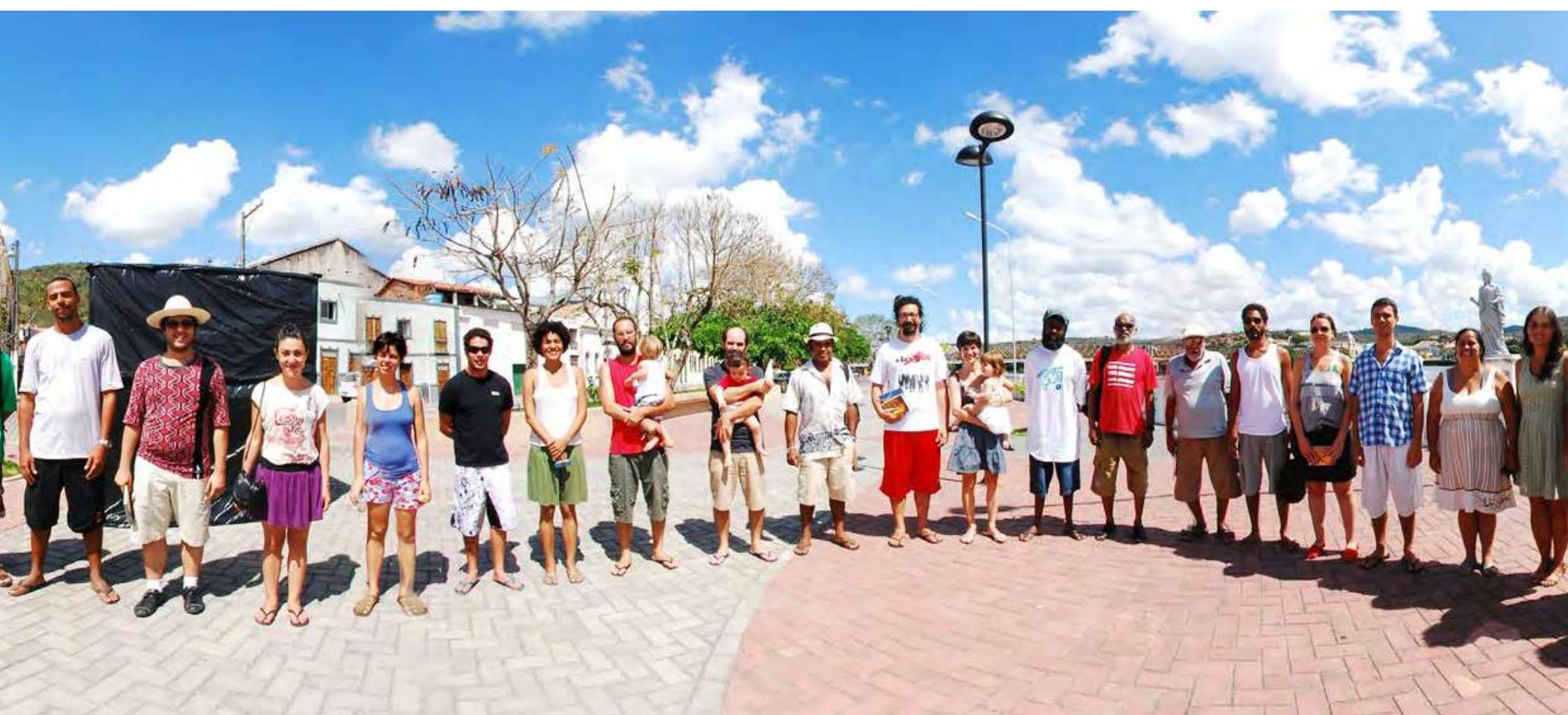


mapeo de distintas problemáticas de Río de Janeiro, respecto al control social, procesos de gentrificación y transformaciones que vive la ciudad y de cómo, a partir de la toma de consciencia de estas transformaciones, se ha ido vinculando a diferentes organizaciones activistas. Nos contó acerca del grupo In.sur.rei.ção del cual formaba parte y su trabajo en conjunto al activista español Pablo de Soto y la activista italiana Laura Burocco. Ahondó en la importancia de Fiar, como una instancia que traslada dinámicas de redes virtuales a un espacio de encuentros presenciales.

Como una ofensiva al control social en nuestras ciudades, como estrategia irónica de insurrección al problema de la falta de espacios de recreación y libertad, a la ciudad cooptada por el capital, la gentrificación y la especulación inmobiliaria y como festividad colectiva, participamos de la intervención "Descascando o abacaxi", organizada por OPA! + GIA, que junto a Felipe Brait nombramos como una acción de "errorismo tropical" 22. En una deriva desde Santo Antônio hasta la Rótula do Abacaxi, en un clima distendido, de celebración, junto al "carrinho de bebê" de OPAVIVARÁ! y el "carrinho SambaGia", estuvimos bailando, cantando, bebiendo, comiendo y haciendo del deseo de estar juntos, la solidaridad social y la pertenencia algo vivo y real. La "Rótula do Abacaxi" o "la Rotonda de la Palmera" fue el espacio escogido por los colectivos como símbolo de un absurdo en la arquitectura de la ciudad: la rotonda está llena de palmeras que no pueden seguir creciendo porque sobre ellas se ha construido una gran autopista.

\section{Etapa 8 - Sao Paulo - Contradicciones, marginalidad, grupos sociales complejos.}

Después de Bahía, continué hacia Sâo Paulo, con una Residencia Temporal en casa de Felipe Brait, con quien veníamos generando fuertes lazos afectivos y profesionales desde 2008, a causa de dos residencias temporales que realizó en mi casa ${ }^{23}$ y una serie de intercambios que fuimos generando en el tiempo.

Paseando por la ciudad, tuvimos un encuentro casual con Flavia Vivacqua articuladora de la red Corocoletivo ${ }^{24}$. La conversación con Flavia fue crucial para tomar consciencia de ciertas ideas respecto a la excesiva proliferación de redes colaborativas que emergen en la América Latina actual. Flavia, con su amplia trayectoria en diversos colectivos de arte, organizaciones sociales y redes, nos aclaró estas ideas: toda red que emerge, joven y voraz, comienza con el deseo de 
abarcar a las prácticas colaborativas como un todo. Sin embargo, tanto Flavia como nosotros, con el paso del tiempo, nos hemos ido dando cuenta que a partir de la heterogeneidad de las prácticas colaborativas existe una dificultad de pensarlas de forma global. Y que la importancia en tanto activistas, no es componer una construcción reticular global de Latinoamérica, sino afianzar espacios tanto virtuales como presenciales de "calidad".

Corocoletivo es también un ecosistema tropical, es decir, "estructuras dentro de estructuras". Como miembros del YahooGroup ${ }^{25}$ desde 2008, hemos constatado que en medio de esta red circula y se intercambia el más heterogéneo flujo de información: desde convocatorias y subvenciones de arte, luchas socio-ambientales, discusiones filosóficas, éticas y estéticas, intercambio de información sobre software y cultura libre, promoción de actividades culturales, trueque de productos y servicios, textos, revistas impresas y virtuales sobre cultura, diálogos sobre ecología, sustentabilidad y permacultura, luchas de defensa animal, luchas por la dignidad de pueblos originarios de Brasil o en contra del racismo, problemáticas de género y disidencia sexual, fotos y videos sobre intervenciones urbanas, encuentros, ofertas de trabajo relacionadas con arte y cultura, pedagogía o informática, difusión de cineclubs, iniciativas barriales, actividades e iniciativas de colectivos, información sobre redes, comunidades afro-brasileñas o sobre grupos en defensa de víctimas de luchas armadas que suceden al interior de Brasil, y un largo etcétera.

\section{Palabras finales}

Procesando las múltiples experiencias del viaje, ya en Chile, algunas conclusiones que surgieron fueron: pensar el trabajo colaborativo/en red en nuestra región como un ecosistema tropical complejo significa comprender que al interior de cada contexto "las estructuras dentro de estructuras" están diferenciadas por aspectos sectoriales existentes en cada país. Y que si bien nos atraviesan problemáticas transversales, a partir de estas divisiones podemos establecer múltiples categorías y sub-categorías de organizaciones que trabajan en red, respecto a las políticas, poéticas, necesidades y estrategias de cooperativismo en que están basadas. Tras el trabajo colaborativo/en red, existen nuevos deseos y formas de experimentar aquello que los pueblos originarios del continente siempre han practicado: el trabajo en comunidad como una instancia que contiene en sí mismo un poder curativo y como práctica de desalienación. 


\section{Referencias}

BEY, Hakim, T.A.Z, Zona autónoma temporal (Colección Nómada\#1). Barcelona: Editorial Anagal, 2007.

ROLNIK, S.; GUATTARI, F. Micropolítica. Cartografías del Deseo (Colección Mapas). Madrid: Traficantes de Sueños, 2006.

SOLÉ, Ricard. Redes Complejas. Del genoma a internet (Colección Metatemas). Barcelona: Tusquets Editores, 2009.

TRANSDUCTORES. Transductores: Pedagogías Colectivas y Políticas Espaciales. (publicación de Transductores en conjunto con el Centro José Guerrero de Granada, Aulabierta, y coproducido por la Universidad Internacional de Andalucía-UNIA arteypensamiento y el Ministerio de Cultura. España). 2009. Disponible en:

http://blogcentroguerrero.org/wp-content/uploads/2014/02/transd-completo-10feb.pdf

ZIBECHI, Raúl. Autonomías y emancipaciones: América Latina en movimiento. Lima: Fondo Editorial de la Facultad de Ciencias Sociales, Universidad Nacional Mayor de San Marcos, 2007.

\section{Referencias web}

Sitio web de FIAR 3 - https://fiarbahia.wordpress.com

Fan Page de Irmandade da Boa Morte - https://www.facebook.com/ IrmandadedaBoaMorte

Sitio web de Casa da Alegría - http://tininhallanos.wordpress.com

Sitio web de Residencia Temporal - http://residenciatemporal.blogspot.com

Sitio web de Ecosistema Tropical - http://www.ecossistema.art.br

Blog de GIA - http://www.giabahia.blogspot.com

Web de Ricardo Brazileiro - http://rbrazileiro.info

Web de N-1 - https://n-1.cc

Entrevista a Milena Durante realizada por el equipo de registros de FIAR - https://www.youtube.com/watch?v=qSLhQxx2qzY

Entrevista a Ricardo Brasileiro - https://www.youtube.com/watch?v=In1luXJ8OBO

Video de la "Palestra Show" https://www.youtube.com/watch?v=GWyL8L-X3t0

Acción de Mucambo Nuspano - https://www.youtube.com/watch?v=GJVj-0SeVII

Información sobre CAMBANA - https://cambana.wordpress.com

Sitio web de Frente 3 de Fevereiro - http://www.frente3defevereiro.com.br

Sitio web de OPAVIVARÁ! - http://www.opavivara.com.br

Fan Page de XØKE :: Mostra Independente de Arte de Guerra - https://www.facebook.com/mostraxoke

\section{Notas}

1 Este texto es una adaptación de varios escritos realizados anteriormente, entre ellos una trilogía de textos llamada "Ecosistema Tropical 2.0: esbozo sobre un nuevo hábitat posible" publicados en la revista Arte y Crítica en 2012, el texto que lleva el mismo nombre que este en el catálogo de FIAR 3 y textos que componen la cartografía impresa sobre colectivos de intervenciones urbanas en Brasil realizada durante el proyecto Ecossistema Tropical 2.0 en 2016. Está centrado en los meses de febrero y marzo durante mi estadía en Brasil y la participación en diferentes encuentros con colectivos brasileños. 
2 Desislaciones fue una plataforma que tuvo por objetivo investigar, difundir y vincular a iniciativas culturales, organizaciones, colectivos, espacios y programas de residencias artísticas que funcionaban en Latinoamérica. El proyecto fue albergado durante 2006 a 2011 en distintos blogs y el sitio web www.desislaciones.net que actualmente no existe.

3 Para más información ver sitio web del festival https://fiarbahia.wordpress.com

4 Descripción oficial de Casa da Alegría: “Projeto livre para construção de um espaço - residência - utilizando pensamentos em tecnologia, arte, comunicação, ecologia, sustentabilidade, liberdade, música, design, acessibilidade, amorosidade... Localizado no litoral norte baiano, pretende se tornar em um espaço experimental, onde o processo seja valorizado por multirões e coletividades itinerantes." Extraída de http://tininhallanos.wordpress.com

5 Sitio web del proyecto: http://residenciatemporal.blogspot.com

6 Para más información visitar: www.ecossistema.art.br

7 "Mucambo Nuspano es un colectivo que trabaja la moda a partir de la identidad de matriz africana. En FIAR desarrollaron una performance donde trabajaron fotografía, graffiti, moda e identidad". Texto extraído del video FIAR\#3 disponible en: https://www.youtube. com/watch?V=GJVj-OSeVII

8 Existe una entrevista a Milena Durante realizada por el equipo de registros de FIAR que contextualiza la iniciativa disponible en: https://www.youtube.com/watch?v=qSLhQxx2qzY

9 Artista e investigador de arte electrónico, vinculado a diferentes organizaciones que trabajan con software libre en Brasil y Latinoamérica y participó en el 2011 de LabSurLab Medellín. Más información del artista en: http://rbrazileiro.info

10 Descripción extraída de la web www.fiarbahia.wordpress.com. Entrevista a Ricardo Brasileiro donde explica el proyecto disponible en: https://www.youtube.com/watch?v=In1/uXJ8OBQ

11 Video donde se puede ver la "Palestra Show" https://www.youtube.com/watch?v=GWyL8L-X3t0

12 Proyecto coordinado por Cristiano Figueiró en Puerto Alegre a partir del cual se creó un CD con música de Mestres que desde la década del 60 realizaban producciones autorales independientes.

13 Descripción extraída del blog de Fiar, disponible en https://fiarbahia.wordpress.com/as-intervencoes

14 Más información en https://cambana.wordpress.com

15 Entrevista a Everton Marco y parte de la acción, disponible en: https://www.youtube.com/watch?v=nNdeE_DPUx4

16 "Anillo sur es un semilla de Lorea, una herramienta libre para redes sociales de América Latina. Es una comunidad de personas preocupadas por la seguridad, la privacidad y mantener el control de las herramientas de comunicación que utilizamos y los datos que compartimos en ellas." Información extraída de: http://media.espora.org/mgoblin_media/media_entries/1122/manual-anillo_sur.pdf

17 Información aportada por Capo, Héctor Capossiello (Chile), hacker, mediactivista y parte del grupo de administradores de N-1 y AnilloSur.

18 Para más información en el sitio del proyecto: https://n-1.cc

19 “TRANSDUCTORES es un proyecto cultural que incluye la puesta en marcha de seminarios y talleres de formación, la construcción y exposición de un archivo relacional, el trabajo con agentes locales y la edición de diversas publicaciones". (TRANSDUCTORES, 2009, p. 2) 
20 Para más información en sitio oficial del colectivo http://www.frente3defevereiro.com.br

21 En el sitio web de OPA! podemos ver el proceso de trabajo que llevaron a cabo ambos colectivos, disponible en http://www.opavivara.com.br/p/me-de-motivos--opa-gia/me-de-motivos--opa-gia

22 En honor a la internacional errorista. www.elinterpretador.net/22Etcetera-Errorismo.html

23 Ver más sobre la experiencia de Felipe Brait en Barcelona en http://residenciatemporal.blogspot.cl/2008/12/felipe-brait-en-residencia-temporal-en.html

24 Más información sobre el trabajo de Flavia Vivacqua y el proyecto CoroColetivo en https://flaviavivacqua.wordpress.com

25 Lista de correo de Coro Coletivo: corocoletivo@yahoogrupos.com.br

26 A XØKE :: Mostra Independente de Arte de Guerra é uma mostra independente, provocada pelo GRUPO ETC para intervir e desestabilizar a coreografia imposta na cidade. Espaço para corpos, gestos, gritos, imagens, impulsos, repúdios, transições, transmutações, provocações e o que servir no combate contra o 'cistema' e suas paredes, grades, correntes e forcas impostas. Información extraída de https://www.facebook.com/mostraxoke. 